ARTICLE

\title{
An optimized procedure for plant recovery from somatic embryos significantly facilitates the genetic improvement of
} Vitis

\author{
Zhijian T Li ${ }^{1}$, Kyung-Hee Kim ${ }^{1}$, Sadanand A Dhekney ${ }^{2}$, Jonathan R Jasinski ${ }^{1}$, Matthew R Creech ${ }^{1}$ and Dennis J Gray ${ }^{1}$
}

Plant regeneration from grapevine (Vitis spp.) via somatic embryogenesis typically is poor. Recovery of plants from Vitis rotundifolia Michx. (muscadine grape) is particularly problematic due to extremely low efficiency, including extended culture durations required for embryo-plant conversion. Poor plant recovery is an obstacle to the selection of improved genetically modified lines. Somatic embryos (SEs) of V. rotundifolia cultivar Delicious (Del-HS) and Vitis vinifera L cultivar Thompson Seedless (TS) were used to identify culture media and conditions that promoted embryo differentiation and plant conversion; this resulted in a two-step culture system. In comparative culture experiments, C2D medium containing $6 \%$ sucrose was the most effective, among four distinct formulae tested, for inducing precocious SE germination and cell differentiation. This medium, further supplemented with $4 \mu \mathrm{M} 6$-benzylaminopurine (C2D4B), was subsequently determined to enhance post-germinative growth of SE. MS medium supplemented with $0.5 \mu \mathrm{M}$ 1-naphthaleneacetic acid (MSN) was then utilized to stimulate root and shoot growth of germinated SE. An average of $35 \%$ and $80 \%$ 'Del-HS' and 'TS' SE, respectively, developed into plants. All plants developed robust root and shoot systems and exhibited excellent survival following transfer to soil. Over 150 plants of 'Del-HS' were regenerated and established within 2.5 months, which is a dramatic reduction from the 6- to 12-month time period previously required. Similarly, 88 'TS' plant lines were obtained within the same time period. Subsequently, seven out of eight Vitis cultivars exhibited significantly increased plant conversion percentages, demonstrating broad application of the two-step culture system to produce the large numbers of independent plant lines needed for selection of desired traits.

Horticulture Research (2014) 1, 14027; doi:10.1038/hortres.2014.27; published online: 11 June 2014

\section{INTRODUCTION}

Cultivars of Vitis rotundifolia Michx. (muscadine grape) are widely grown in the southeastern United States, where they are utilized for fresh fruit, juice, jam and wine. ${ }^{26}$ Important health benefits associated with the fruit of $V$. rotundifolia include high content of dietary fiber, resveratrol, phenolics and other antioxidants with anti-inflammatory and anticancer properties. ${ }^{9,12,14,15,23,24}$ Development of cultivars with genetically improved traits, such as better postharvest storage and seedlessness, as well as other quality and yield attributes, is needed to expand the $V$. rotundifolia fruit industry in the southeastern United States. ${ }^{2}$

$V$. rotundifolia is a member of the Vitis subgenus Muscadinia Planch. Species of Muscadinia contain a chromosome number of $40(2 n)$ compared to that of subgenus Euvitis Planch $(2 n=38)$, which includes the economically important bunch grape cultivars derived from $V$. vinifera $L$. and hybrids. Thus, it is particularly difficult to introgress useful genes/traits from bunch grape to muscadine grape by way of breeding. ${ }^{6}$ For instance, transfer of the seedless trait from $V$. vinifera to $V$. rotundifolia to produce commercially useful seedless muscadine grape cultivars has not yet occurred, despite concerted breeding efforts for more than half of a century.

Genetic engineering technology facilitates the introduction of single-gene traits without altering existing agronomic performance; thus, it provides an attractive tool to overcome genetic constraints and expedite progress for Vitis improvement. However, successful implementation of the technology largely hinges on the ability to obtain plants from engineered somatic embryos (SEs) and requires an efficient and reliable plant regeneration system. ${ }^{7}$

Despite relatively low SE-to-plant recovery overall, advances in genetic engineering technology for cultivars of Euvitis have resulted in a large number of transgenic grape plants for genetic, molecular and functional studies. ${ }^{1,3,17,19,28,29}$ In comparison, similar technological developments for Muscadinia have not occurred. Only four investigative reports concerning somatic embryogenesis and plant regeneration have appeared. ${ }^{4,5,18,20}$ Dhekney et al. ${ }^{18}$ extensively characterized the somatic embryogenic culture response of a number of $V$. rotundifolia cultivars and provided an optimized protocol for callus induction, SE production and plant recovery. However, the efficiency of SE-to-plant conversion with $V$. rotundifolia remains lower and more time-consuming than Euvitis, which also typically exhibits poor plant recovery as well.

In this report, we compare several basal media commonly used in grapevine tissue culture to determine their efficacy on SE germination and growth. With timed addition of two plant growth regulators, a two-step culture procedure for embryo germination and root induction, respectively, was developed to significantly improve plant recovery from SE of both Muscadinia and Euvitis cultivars.

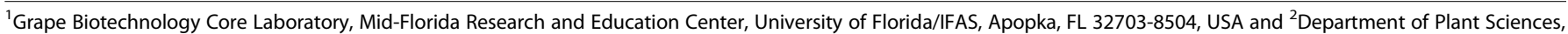
Sheridan Research and Extension Center, Sheridan, WY 82801, USA

Correspondence: Correspondence: DJ Gray (djg@ufl.edu)

Received: 8 April 2014; revised: 11 April 2014; accepted: 15 April 2014 


\section{MATERIALS AND METHODS}

Chemicals and medium preparation

Chemicals for the preparation of culture media were acquired from Fisher Scientific (Pittsburgh, PA, USA) and Sigma-Aldrich Co. (St Louis, MO, USA). TC agar was purchased from Phytotechnology Laboratories, LLC (Shawnee Mission, KS, USA). Four media used for comparing SE maintenance are (i) basal salts and vitamins from DM medium, ${ }^{27}$ which is a modified DKW medium developed by Driver and Kuniyuki; ${ }^{10}$ (ii) MS medium; ${ }^{25}$ (iii) C2D medium; ${ }^{16}$ and (iv) $\mathrm{X} 6$ medium. ${ }^{27}$ Six percent ( $\mathrm{w} / \mathrm{v}$ ) sucrose was added to each basal medium resulting in D6, M6, C6 and X6 media, respectively.

To study SE germination and plant recovery, the following previously described media were compared: (i) C2D4B (C2D medium supplemented with $3 \%(\mathrm{w} / \mathrm{v})$ sucrose and $4 \mu \mathrm{M}$ 6-benzylaminopurine); (ii) MS1B (MS medium containing $3 \%(\mathrm{w} / \mathrm{v})$ sucrose and $1 \mu \mathrm{M}$ benzylaminopurine); and (iii) MSN (MS medium containing 3\% (w/v) sucrose and $0.5 \mu \mathrm{M}$ 1-naphthaleneacetic acid). All culture media were adjusted to $\mathrm{pH} 5.8$ with a $1 \mathrm{~N} \mathrm{KOH}$ solution and supplemented with $7 \mathrm{~g} \mathrm{~L}^{-1} \mathrm{TC}$ agar prior to autoclaving at $121^{\circ} \mathrm{C}$ for $20 \mathrm{~min}$. Cooled medium was dispensed onto Petri dishes $\left(30 \mathrm{~mL}^{\text {dish }}{ }^{-1}\right)$ or pre-sterilized Magenta GA7 vessels (Sigma-Aldrich) $\left(70 \mathrm{~mL}\right.$ vessel $\left.{ }^{-1}\right)$.

\section{Plant material}

SE from two V. rotundifolia cultivars including Alachua (Ala) ${ }^{11}$ and Delicious $\left(\right.$ Del) ${ }^{8}$ plus a genetically modified line of Delicious (Del-HS) ${ }^{20}$ four $V$. vinifera cultivars including Chardonnay (Cha), Chenin Blanc (Che), Merlot (Mer), Thompson Seedless (TS) and one V. vinifera hybrid cultivar, Seyval Blanc (Sey) were employed. These cultivars previously were studied for their performance in producing the required somatic embryogenic cultures for genetic transformation. ${ }^{19,27,28}$ In investigative experiments to increase plant recovery, only SE of 'TS' and 'Del-HS' were utilized. The remaining six cultivars were tested to confirm utility of the resulting improved culture procedure across Vitis genotypes.
Somatic embryogenesis

SE were initiated from immature leaf explants of in vitro shoots ${ }^{18}$ or stamens ${ }^{21}$ and maintained by subculturing proembryonic masses on X6 medium at monthly intervals. ${ }^{21,22}$

To compare SE maintenance on each basal medium, a total of 15 SEs were combined into a group (replicate), with four groups per dish and three dishes per medium treatment (i.e., 12 replicates). Eight treatments included SE at torpedo or early cotyledonary stages of development, each tested on the four previously mentioned maintenance media. Cultures were kept in the dark for a total of 2 months with monthly subculture to fresh medium prior to data collection for explant fresh weight, total number of SE produced and frequency of precocious SE germination.

\section{SE germination and conversion to plants}

To test SE germination and plant regeneration, four SE at mid-cotyledonary stage of development were placed in each GA7 vessel on one of the four previously-mentioned germination/plant development media with 10 replicate vessels per treatment. Cultures were maintained under cool white fluorescent light $\left(65 \mu \mathrm{m} \mathrm{m}^{-2} \mathrm{~s}^{-1}\right.$ and a 16-h photoperiod) at $26{ }^{\circ} \mathrm{C}$. The growth of shoots and roots were monitored 3 weeks after culture initiation via measurement of fresh weight of individual SE. Germinated SE were then moved to MSN medium for root induction cultivation for 4 weeks. Rooted plants were transferred to potting soil in $3^{\prime \prime}$ pots and acclimatized in a mist bed for 1 week before being moved to a greenhouse bench.

The optimum duration of SE culture on C2D4B medium for plant development was determined by placing 'Del-HS' SE on C2D4B medium for 1, 2, 3 and 4 weeks, respectively, followed by subculture onto MSN medium. The resulting number of plants was determined for each treatment 1 month after culture on MSN medium. All experiments were repeated two times. Data were collected and statistically analyzed to yield mean values and standard errors for assessment of sample variation

\section{Somatic Embryogenic Culture and Genetic Modification System for Grape}
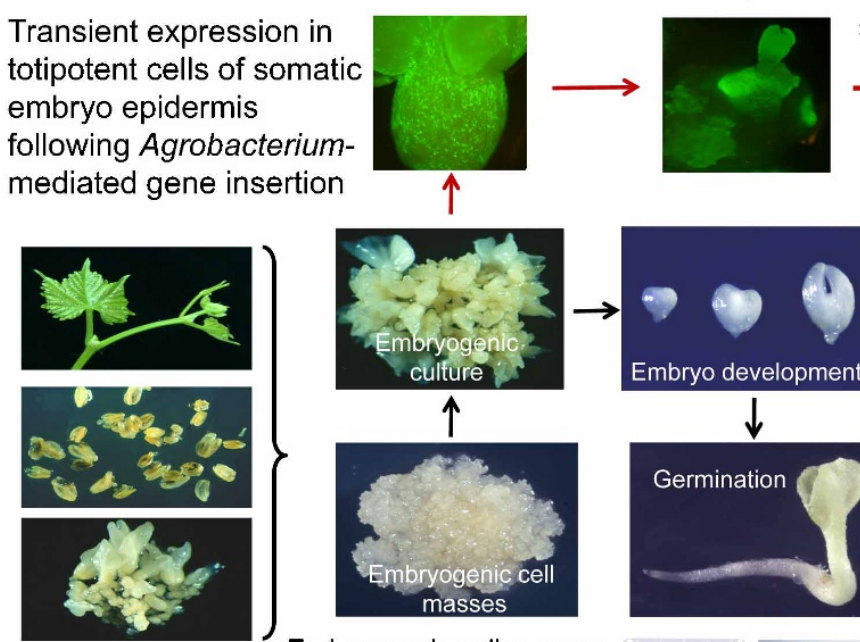

Explants

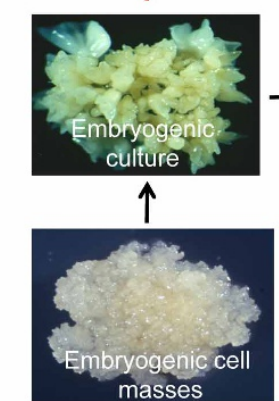

Embryogenic cell masses induced from various explants produce somatic embryos, which germinate and develop into plants.
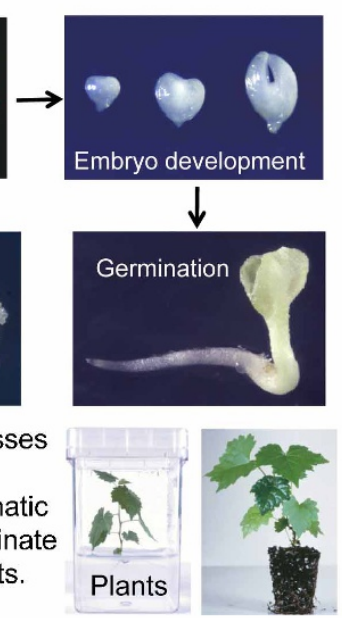

Stable expression from selected embryogenic cell cultures

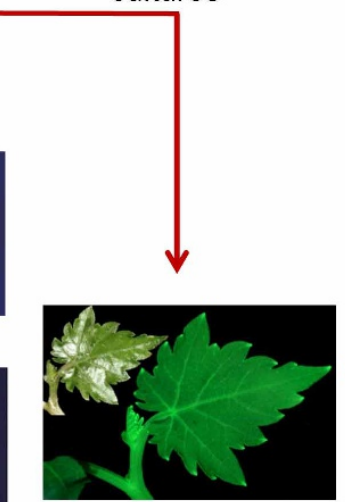

\section{Genetically-modified} grape plants

Figure 1. Visual representation of the somatic embryogenesis system for grape. Depending on grape genotype, several different types of explants may be utilized to initiate embryogenic cultures, including shoot tips and leaves, stamens or pre-existing SEs. Embryogenic cell masses composed of proembryonal complexes are isolated from callusing explants. Cell masses produce SEs, which can be encouraged to germinate and develop into plants. The germination-to-plant step typically is very poor, which was dramatically improved by this study. It is at the stage of SE maturation (red arrows) that they may be utilized as targets for Agrobacterium-mediated gene insertion due to the abundance of totipotent cells on their hypocotyl and cotyledon surfaces. Utilizing the GFP gene as a visible marker, abundant transient GFP-expressing cells occur. Stable modified embryogenic lines are recovered, from which are obtained non-chimeric, genetically modified plants. GFP, green fluorescent protein. 


\section{RESULTS}

The typical procedure to initiate embryogenic cultures, which is used to produce plants, is illustrated in Figure 1. The parallel development of genetically modified plants (red arrows) was illustrated by using a visible green fluorescent protein marker. A key obstacle in the SE-to-plant process is the step from SE germination to whole plant development, which typically restricts the number of plants obtainable from a given genetically modified plant line.

Effects of basal medium on SE maintenance

Four basal media including D6, M6, C6 and X6 without growth regulators but with $6 \%(W / V)$ sucrose were examined for SE proliferation and growth of both $V$. rotundifolia and $V$. vinifera cultivars. These media containing ingredients at distinctively different concentrations (Supplementary Fig. S1) are commonly used for grapevine tissue culture, ${ }^{16,27,28}$ but had not been evaluated in a comparative test. Culture of SE of both 'Del-HS' and 'TS' on these media resulted in dramatic differences in SE proliferation and precocious SE germination as signified by enlargement, greening, hypocotyl elongation and root development (Supplementary Fig. S2).

SE proliferation also varied between the two cultivars and among media. In general, SE explants of 'Del-HS' displayed higher secondary SE proliferation than 'TS'. Among the compared media, D6 medium was most effective for SE proliferation, giving rise to 120 and 60 SEs per group for 'Del-HS' and 'TS', respectively (Figure 2a). Both $\mathrm{M} 6$ and $\mathrm{X} 6$ media performed similarly but with slightly lower SE proliferation. Noticeably, C6 medium led to the lowest total number of SE per group for both cultivars (Figure 2a).

A higher percentage of 'TS' SE precociously germinated on all media when compared with 'Del-HS'. On D6 medium, about $20 \%$ of 'Del-HS' SE underwent precocious germination compared to that of 'TS' SE, which reached almost $60 \%$ (Figure $2 \mathrm{~b}$ ). A similar trend was observed on $\mathrm{M} 6$ medium with a slightly increased germination rate for both cultivars. Up to $60 \%$ and $100 \%$ of SE from 'Del-HS' and 'TS', respectively, germinated on C6 medium. On X6 medium, germination of 'Del-HS' SE was less than $10 \%$, whereas that of 'TS' SE was about $50 \%$ (Figure 2b). C6 medium stimulated the greening of $\mathrm{SE}$ even in dark-grown cultures (Supplementary Fig. S2). Thus, C6 medium is more conducive to precocious SE germination and cell differentiation/greening, while providing the least favorable conditions for SE proliferation.

SE germination and plant development

Based on aforementioned findings of growth responses of SE cultured on different basal media in darkness, we further investigated SE germination and seedling development under light on various media, including plant growth regulator-containing media C2D4B, MS1B, and MSN. These media are commonly used for micropropagation and plant recovery of Vitis. ${ }^{21,22,28}$

Three weeks after culture initiation, all SE explants germinated on all three media. SEs of both 'Del-HS' and 'TS' expanded dramatically in size when cultured on C2D4B medium (Figure 3a, C2D4B). In particular, the cotyledons turned dark green and became oversized and thickened. Noticeably, some 'TS' SE developed robust roots and enlarged greenish cotyledons. On the other hand, no roots were produced by 'Del-HS' SE (Figure 3a, C2D4B, lower dish vs. upper dish). On MS1B medium, similar size expansion of cotyledons was evident for 'TS' SE but to a significantly lesser degree for 'Del-HS' SE (Figure 3a, MS1B, lower dish vs. upper dish). These findings are in agreement with observations of reduced cotyledon expansion in germinated SE of $V$. rotundifolia cultivars as compared to $V$. vinifera under similar medium conditions. ${ }^{18}$ Although root development occurred from SEs of both cultivars on MS1B, roots from 'Del-HS' seedlings were longer in length, smaller in diameter and branched while those from 'TS' embryos were thicker in diameter and shorter
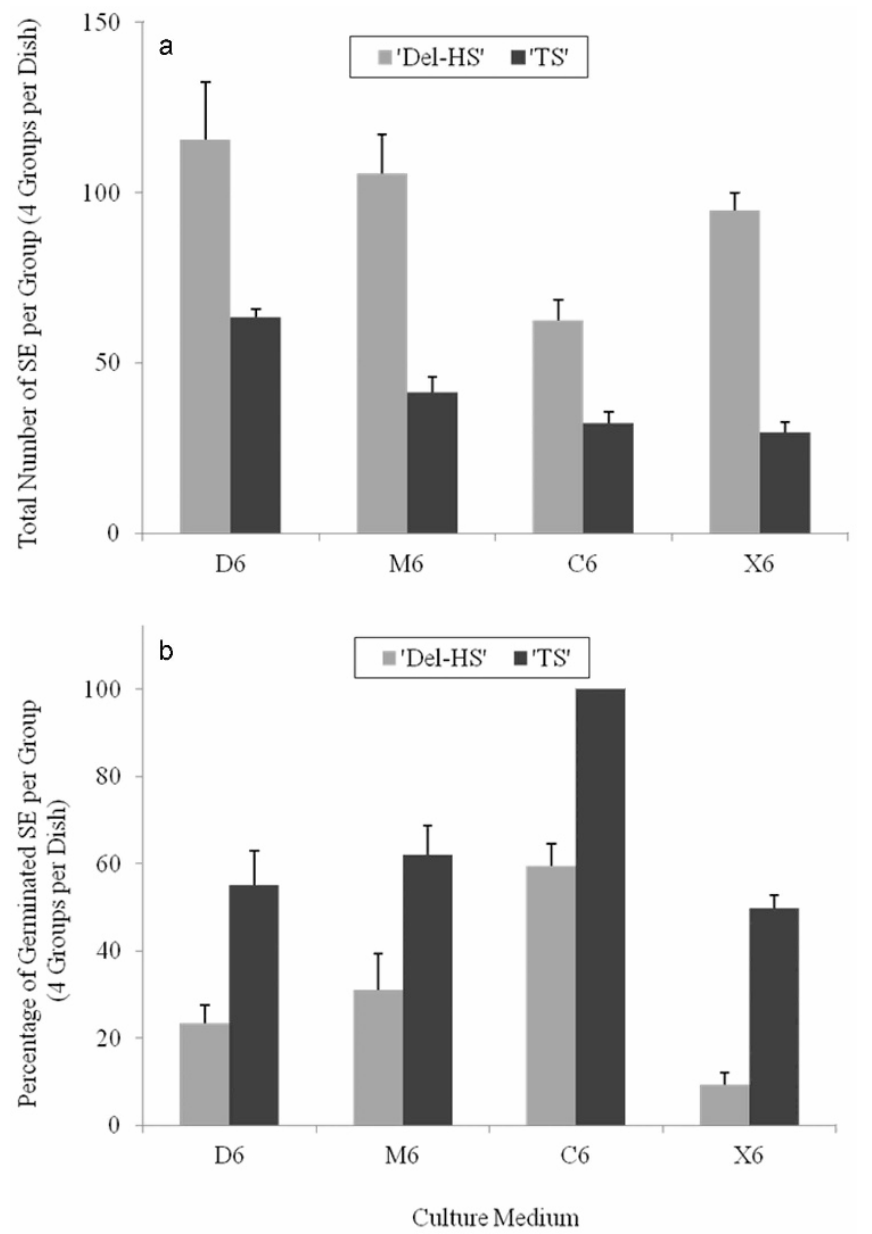

Figure 2. SE proliferation and germination after 2-month culture on various basal media. (a) The total number of resulting SE per group was determined. Bar values represent the averaged number of resulting SEs per group for each cultivar including both explant sizes. Standard errors were indicted. (b) Germination frequencies of SEs per group were evaluated. Data represent averaged percentage for each cultivar from all explants including both small and large sizes. Standard errors were indicted.

in length without lateral branching (Figure 3a, MS1B, upper dish vs. lower dish). These experiments exemplified the relatively weak vegetative growth of SE-derived plants of $V$. rotundifolia on MS1B medium. All SEs of 'Del-HS' and 'TS' cultured on MSN were smaller and had very weak-to-no root systems (Figure 3a, MSN).

To quantify growth, the fresh weight of individual SE was measured at the end of a 3-week culture period. The fresh weight of 'DelHS' SE cultured on three media illustrated a 6.3- and a 2.2-fold increase on C2D4B and MS1B, respectively, when compared to MSN (Figure 4, top panel). Fresh weight for 'TS' SE increased 3.1and 2.2-fold on C2D4B and MS1B, respectively, when compared to MSN (Figure 4, bottom panel).

\section{Plant recovery from a two-step culture system}

To investigate whether improved root growth could break the stagnation of shoot development, SE germinated on different media for 3 weeks were subsequently transferred to 1-naphthaleneacetic acid containing MSN medium that was previously employed for root induction from transgenic tobacco shoots. ${ }^{13}$ Noticeably, from previous observations, MSN medium supported only very weak overall growth of 'Del-HS' SE, but did induce roots with larger diameter (Figure 3a, MSN). 

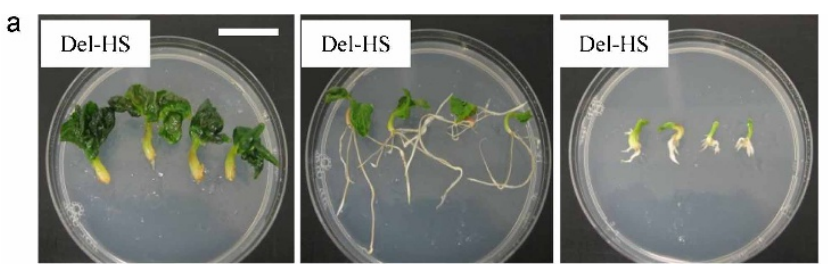

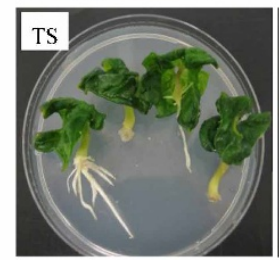

$\mathrm{C} 2 \mathrm{D} 4 \mathrm{~B}$

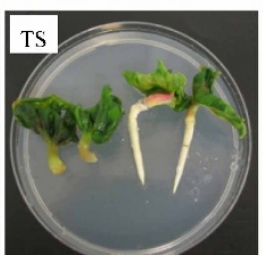

MS1B

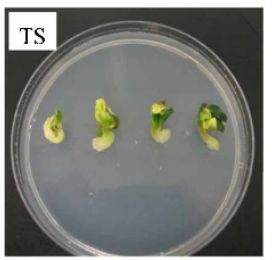

MSN
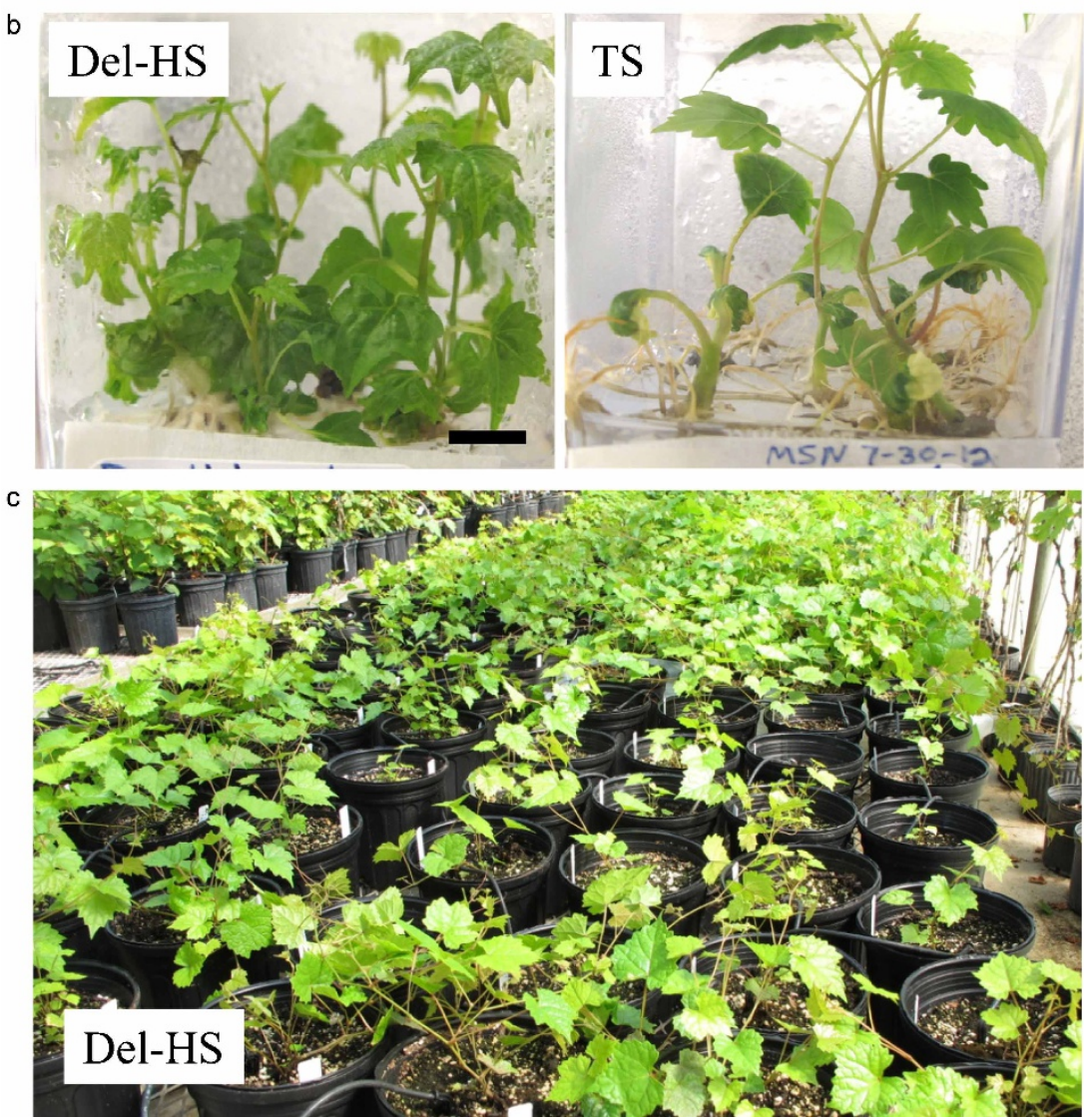

Figure 3. SE germination and plant development using a two-step culture procedure. (a) SE post-germinative growth on various germination media. Bar $=3 \mathrm{~cm}$. (b) Plantlets obtained after culture first on C2D4B medium and then MSN medium in GA7 vessels. Bar marks an actual length of $3 \mathrm{~cm}$. (c) A large number of transgenic 'Del-HS' plants established in the greenhouse. Plants in background on the adjacent bench were SEderived 'TS' plants.

As illustrated in Figure 3b, 4 weeks after transfer to MSN medium, 'Del-HS' SE previously cultured on C2D4B medium had developed into plants with robust shoots and roots. SE explants with such rapid plant development reached 35\% (Figure 5, top panel). In comparison, only $2 \%-4 \%$ of SE explants cultured only on MSN medium without the C2D4B pre-treatment developed into plants. In agreement with previous observations, no in vitro plants were obtained when SE were cultured on MS1B medium (Figure 5, top panel). In comparison, plants of 'TS' were recovered on MSN after pre-treatment of SE on all three media (Figure 5, bottom panel). However, a higher percentage of plant recovery with more vigorous plant growth occurred when SE were previously cultured on C2D4B medium (Figures $3 \mathrm{~b}$ and 5, bottom panel).

Plant recovery from SE as affected by duration on C2D4B Culture duration on C2D4B medium prior to subculture on MSN medium influenced plant development of 'Del-HS' SE. Plant recovery frequencies peaked at 3-week culture on C2D4B medium. Time periods shorter than 2 or longer than 3 weeks reduced plant recovery by almost $50 \%$ (Figure 6). Exposure of SE to C2D4B medium for more than 3 weeks tended to increase the level of tissue hyperhydricity that impeded subsequent plant development (data not shown). 

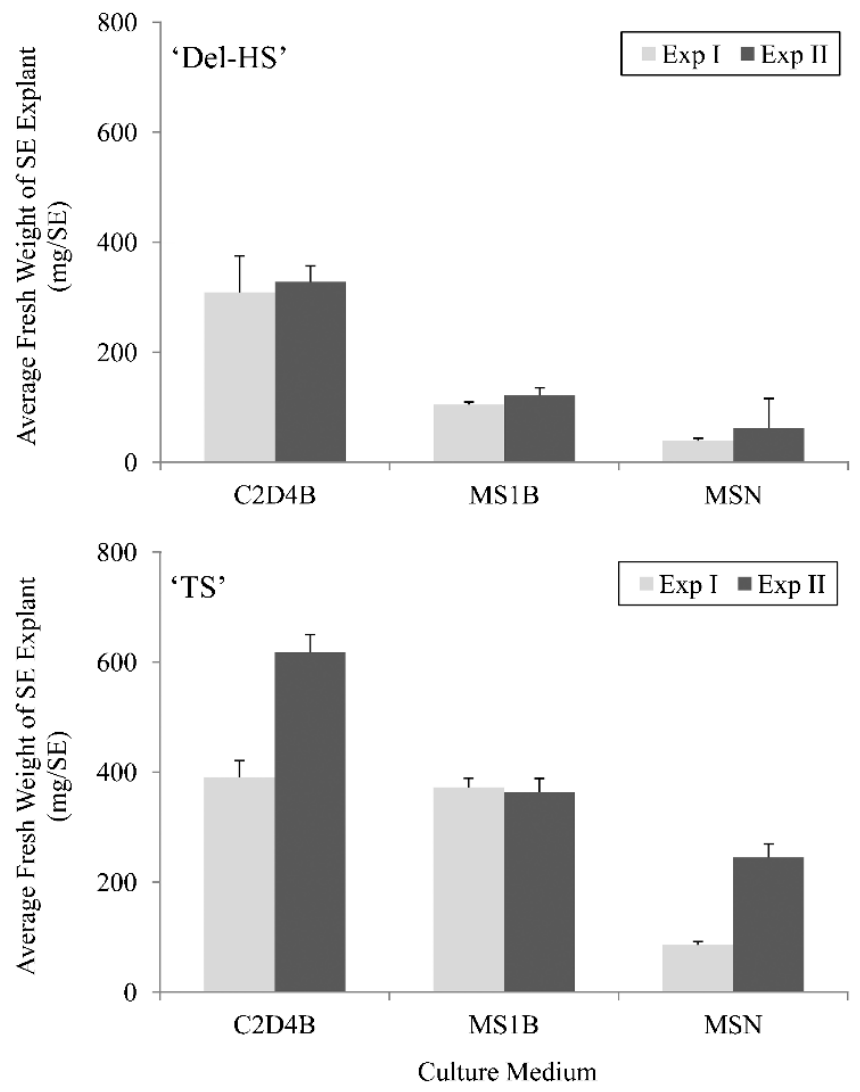

Figure 4. Averaged fresh weight of SE after a 3-week culture on various germination media. SEs at mid-cotyledonary stage of development were placed on test germination medium at four explants per plate/10 plates per medium treatment and maintained under light. Three wk after culture initiation, fresh weight of each SE was determined. Bar values in each panel represent average data for SEs of indicated cultivar on each medium. Lines above data bars denote standard errors.

Plant recovery from SE of various cultivars

General applicability of the best protocol for plant recovery from SE was determined by comparing the optimized two-step culture procedure with the standard MS1B single-medium culture procedure using SE of three $V$. rotundifolia cultivars and lines 'Ala', 'Del', 'Del$\mathrm{HS}^{\prime}$, four $V$. vinifera cultivars including 'Cha', 'Che', 'Mer', 'TS' and $V$. vinifera hybrid 'Sey'. Results showed that seven out of eight tested cultivars/lines had significantly higher frequencies of plant recovery using the two-step procedure (C2D4B-MSN), whereas only one cultivar 'Che' performed better with the standard procedure (Figure 7).

Plant acclimatization

Using the two-step C2D4B-MSN culture procedure, over 150 plants of 'Del-HS' and 88 plants of 'TS' were obtained within 2.5 months. All plants survived transplantation to soil and continuously displayed robust vegetative growth in the greenhouse (Figure 3c). Plants of the other cultivars obtained using the same culture conditions also showed vigorous growth and developed into vigorous plants (data not shown).

\section{DISCUSSION}

In plant tissue culture, genetic milieu of a particular species or genotype often dictates the overall growth response to culture conditions. Vitis rotundifolia is genetically and morphologically different from $V$. vinifera even though both belong to the same genus.

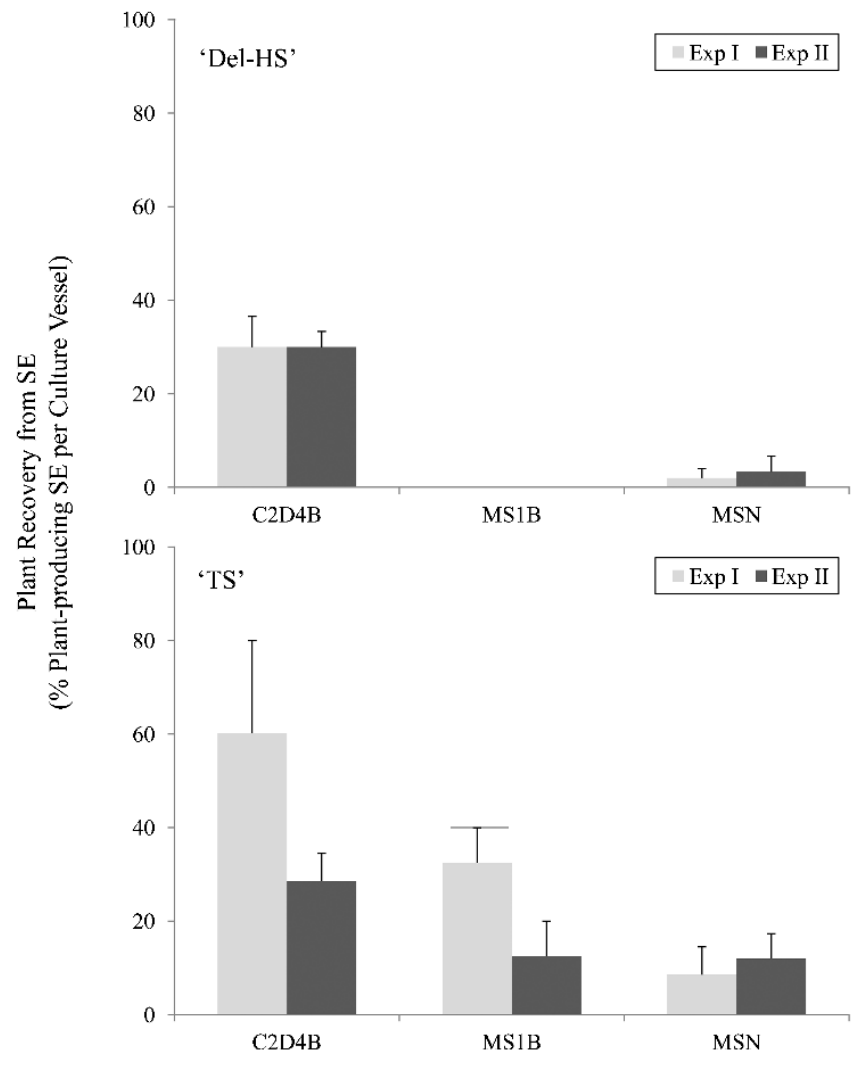

First Step Culture Medium

Figure 5. Plant recovery from SEs cultured first on various germination media and then on MSN medium. SE at mid-cotyledonary stage of development were placed on various germination media at four explants per plate/ 10 plates per medium treatment and maintained under light. After 3 weeks, SE explants were transferred onto MSN medium contained in GA-7 vessels. The percentage of explants developed normal shoots with at least two true leaves in each culture vessel was determined and presented as bar values. Data from two repeated experiments are presented. Lines above each data bar indicate standard errors.

Previous studies showed that $V$. rotundifolia exhibited a difference in embryogenic callus development compared to $V$. vinifera and $V$. rupestris. ${ }^{4,5}$ Somatic embryogenesis and plant regeneration in $V$. rotundifolia displayed unique genotypic responses and required the use of culture media that were different from those used for other grape species. ${ }^{4}$ Although factors affecting callus induction and plant regeneration via somatic embryogenesis in $V$. rotundifolia were characterized recently, ${ }^{18}$ plant recovery frequencies remained low.

In previous studies, germinated SE of Vitis were maintained on the same medium, such as MS1B, with monthly subcultures and removal of cotyledons in order to achieve plant recovery. ${ }^{18,28}$ However, the relatively weak shoot and root systems of $V$. rotundifolia resulted in a long-term culture requirement of up to 1 year, plus poor plant establishment, as exemplified here by 'Del-HS'. Similarly, over several years, less than two dozen transgenic plant lines of $V$. rotundifolia cultivar Alachua were recovered. ${ }^{20}$ Comparatively, within the same time period, over 1500 transgenic plants of $V$. vinifera were generated (unpublished). These varied responses between Muscadinia and Euvitis reflect their distinctive genotypic differences. Lack of an adequate number of genetically engineered plant lines for evaluation of anticipated traits presented a significant obstacle to genetic engineering of $V$. rotundifolia. 


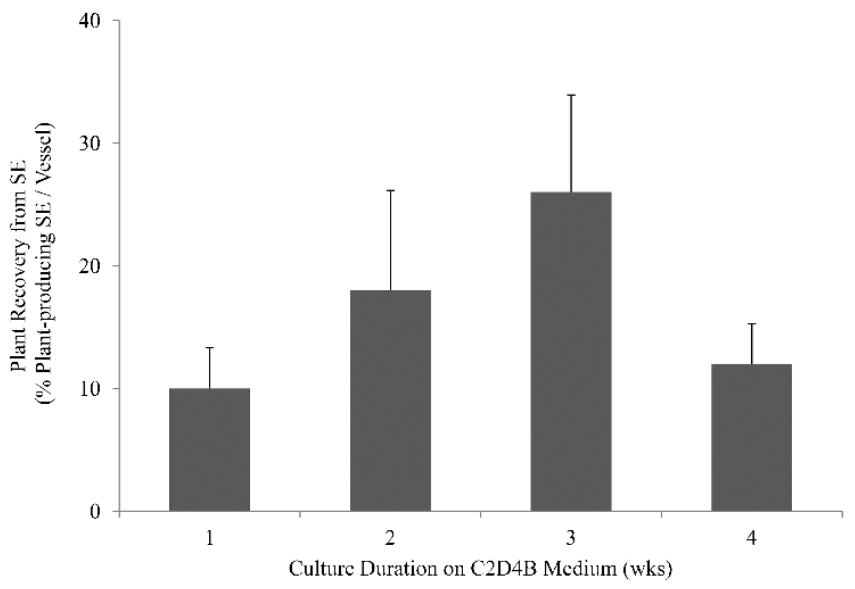

Figure 6. Recovery of genetically modified 'Del-HS' plants influenced by germination culture on C2D4B for varied time periods. SE explants were placed on C2D4B medium and cultured for designated time lengths. Explants were then transferred onto MSN for plant development. Bars represent averaged percentage values of plant recovery from 10 culture vessels with four explants each. Lines above each data point represent standard errors.

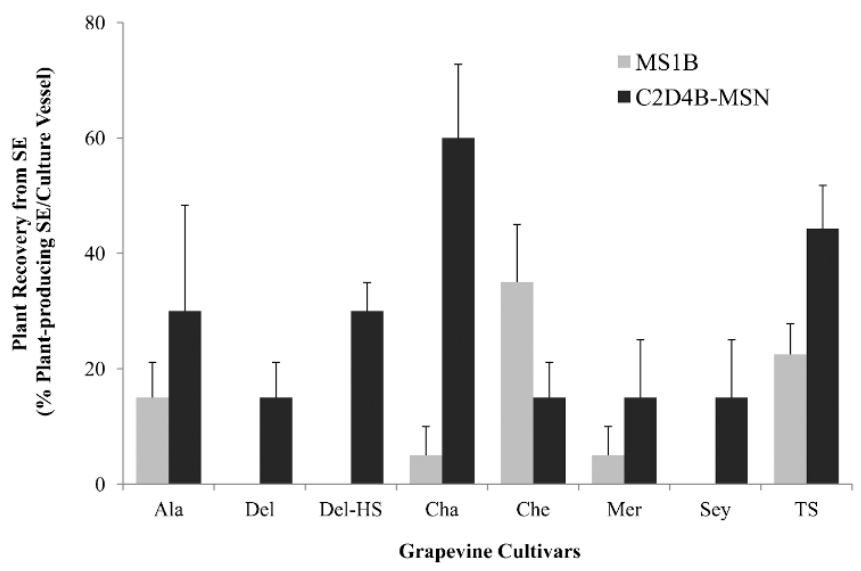

Figure 7. Plant recovery from SE of various cultivars of both muscadine and bunch grapes using the two-step C2D4B-MSN procedure. Cultivar names were described in the section on 'Materials and methods'. Culture procedures followed those detailed in Figure 5. Data represent averaged values from two repeated experiments. Lines above each data bar indicate standard errors.

This study demonstrated the significant improvement of plant recovery from SE of $V$. rotundifolia based on timed application of optimized media. The current procedure dramatically reduces the time required for plant recovery from 6-12 months to 2.5 months. In addition, the resulting plants develop robust and vigorous shoot and root systems and are easily established in the greenhouse. Further, four of five Euvitis cultivars responded positively to this culture procedure.

Enhanced plant recovery from genetically modified SEs facilitates improvement for a wide range of Vitis cultivars. This culture procedure thereby removes a significant obstacle to creating the large numbers of genetically engineered lines of Vitis that are required for the efficient selection of improved cultivars.

\section{CONFLICT OF INTEREST}

The authors declare no conflict of interest.

\section{ACKNOWLEDGEMENTS}

This research was supported in part by the Florida Agricultural Experiment Station, the USDA/NIFA Specialty Crops Research Initiative (grant \# 2011-51181-30668) and the Florida Department of Agriculture and Consumer Services' Viticulture Trust Fund (grant \# 00079907). The authors gratefully thank Ms. Marlene Saldivar and Natalia Cimino of MREC for their excellent technical support.

\section{REFERENCES}

1 Nookaraju A, Agrawal DC. Enhanced tolerance of transgenic grapevines expressing chitinase and B-1,3-glucanase genes to downy mildew. Plant Cell Tiss Organ Cult 2012; 111: 15-28.

2 Alonso AD, O'Neil MA. Muscadine grapes, food heritage and consumer images: implications for the development of a tourism product in Southern USA. Tour Plan Dev 2012; 9: 213-229.

3 Lopez-Perez AJ, Velasco L, Pazos-Navarro M et al. Development of highly efficient genetic transformation protocols for table grape sugraone and crimson seedless at low Agrobacterium density. Plant Cell Tiss Organ Cult 2008; 94: 189-199.

4 Robacker $C$. Somatic embryogenesis and plant regeneration from muscadine grape leaf explants. Hort Sci 1993; 28: 53-55.

5 Gray DJ. Somatic embryogenesis and plant regeneration from immature zygotic embryos of muscadine grape (Vitis rotundifolia) cultivars. Am J Bot 1992; 79: 542546.

6 Gray DJ, Benton. In vitro micropropagation and plant establishment of muscadine grape cultivars (Vitis rotundifolia). Plant Cell Tiss Organ Cult 1991; 27: 7-14.

7 Gray DJ, Dhekney SA, Li ZT et al. Genetic engineering of grapevine and progress towards commercial deployment. In: Mou B, Scorza R (ed.). Transgenic Horticultural Crops: Challenges and Opportunities. Boca Raton, FL: CRC Press, 2011: 317-332.

8 Gray DJ, Li ZT, Dhekney SA et al. 'Del-Hs': an early-ripening self-fertile, multipurpose black-fruited muscadine grape. Hort Sci 2009; 44: 200-201.

9 Pastrana-Bonilla E, Akon CC, Sellappan S et al. Phenolics content and antioxidant capacity of muscadine grapes. J Agric Food Chem 2003; 51: 5497-5503.

10 Driver JA, Kuniyuki AH. In vitro propagation of paradox walnut rootstock. Hort Sci 1984; 19: 507-509.

11 Mortensen JA, Harris JW. Alachua Muscadine Grape. Circular (Florida Agricultural Experiment Station), Alachua County, FL: Agricultural Experiment Station, University of Florida, 1990.

12 God JM, Tate P, Larcom LL. Anticancer effects of four cultivars of muscadine grape. J Med Food 2007; 10: 54-59.

13 Burrow MD, Chlan CA, Sen P et al. High frequency generation of transgenic tobacco plants after modified leaf disk cocultivation with Agrobacterium tumefaciens. Plant Mol Biol Rep 1990; 8: 24-139.

14 Musingo MN, Sims CA, Bates RP et al. Changes in ellagic acid and other phenols in muscadine grape (Vitis rotundifolia) juices and wines. Am J Enol Vitic 2001; 52: 109-114.

15 Greenspan P, Bauer JD, Pollock SH et al. Antiinflammatory properties of the muscadine grape (Vitis rotundifolia). J Agric Food Chem 2005; 53: 8481-8484.

16 Chee R, Pool RM, Bucher D. A method for large scale in vitro propagation of Vitis. NY Food Life Sci Bull 1984; 109: 1-9.

17 Vidal RJ, Rama J, Taboada L et al. Improved somatic embryogenesis of grapevine (Vitis vinifera) with focus on induction parameters and efficient plant regeneration. Plant Cell Tiss Organ Cult 2009; 96: 85-94.

18 Dhekney SA, Li ZT, Gray DJ. Factors influencing induction and maintenance of Vitis rotundifolia Michx. embryogenic cultures. Plant Cell Tiss Organ Cult 2011; 105: 175-180.

19 Dhekney SA, Li ZT, Gray DJ. Grapevine engineered to express cisgenic Vitis vinifera thaumatin-like protein exhibit fungal disease resistance. In Vitro Cell Dev Biol 2011; 47: 458-466.

20 Dhekney SA, Li ZT, Dutt M et al. Agrobacterium-mediated transformation of embryogenic cultures and plant regeneration in Vitis rotundifolia Michx. (Muscadine Grape). Plant Cell Rep 2008; 27: 865-872.

21 Dhekney SA, Li ZT, Compton ME et al. Optimizing initiation and maintenance of Vitis embryogenic cultures. Hort Sci 2009; 44: 1400-1406.

22 Dhekney SA, Li ZT, Zimmerman TW et al. Factors influencing genetic transformation and plant regeneration of Vitis. Am J Enol Vitic 2009; 60: 285-292.

23 Percival SS, Sims CA, Talcott ST. Immune Benefits of Consuming Red Muscadine Wine. Naples, FL: University of Florida Cooperative Extension Service, Institute of Food and Agricultural Sciences, EDIS. Accessed April, 2014 at http://edis.ifas.ufl.edu/fs085

24 Talcott ST, Lee JH. Ellagic acid and flavonoid antioxidant content of muscadine wine and juice. J Agric Food Chem 2002; 50: 3186-3192.

25 Murashige T, Skoog FA. A revised medium for rapid growth and bioassays with tobacco tissue cultures. Physiol Plant 1962; 15: 473-497.

26 Olien WC. Muscadine-a classic southeastern fruit. Hort Sci 1990; 25: 726 831. 
27 Li Z, Jayasankar S, Gray DJ. Expression of a bifunctional green fluorescent protein (GFP) fusion marker under the control of three constitutive promoters and enhanced derivatives in transgenic grape (Vitis vinifera). Plant Sci 2001; 160: 877-887.

28 Li ZT, Dhekney SA, Dutt M et al. An improved protocol for Agrobacterium-mediated transformation of grapevine (Vitis vinifera L.). Plant Cell Tiss Organ Cult 2008; 93 311-321.

$29 \mathrm{Li}$ ZT, Dhekney SA, Dutt M et al. Optimizing Agrobacterium-mediated transformation of grapevine. In Vitro Cell Dev Biol Plant 2006; 42: 220-227. cc)(1) () This work is licensed under a Creative Commons AttributionNonCommercial-NoDerivs 3.0 Unported License. The images or other third party material in this article are included in the article's Creative Commons license, unless indicated otherwise in the credit line; if the material is not included under the Creative Commons license, users will need to obtain permission from the license holder to reproduce the material. To view a copy of this license, visit http:// creativecommons.org/licenses/by-nc-nd/3.0/

Supplemental Information for this article can be found on the Horticulture Research website (http://www.nature.com/hortres). 